\title{
Ações de reorientação da formação profissional em Fisioterapia: enfoque sobre cenários de prática
}

\author{
Ana Paula Maihack Gauer (a) \\ Fátima Ferretti ${ }^{(\mathrm{b})}$ \\ Carla Rosane Paz Arruda Teo (c) \\ Lucimare Ferraz $^{(\mathrm{d})}$ \\ Maria Cristina Flores Soares ${ }^{(e)}$
}

Gauer APM, Ferretti F, Teo CRPA, Ferraz L, Soares MCF. Reorientation of professional education in Physiotherapy: a focus on practice settings. Interface (Botucatu). 2018; 22(65):565-76.

This paper aimed to identify from the perspective of teachers and students, actions that evidence the reorientation of vocational training in physiotherapy happening under the line of practice settings recommended by the Pro-Saude Program. A qualitative research using the case study method, was performed in a Community University, where 16 students and 11 teachers of the Physiotherapy course as participants. Data collection was carried out by focus group and interview. Among the actions that demonstrate the reorientation of vocational training we highlight: achievement the situational diagnosis, practices in growing complexity degrees, diversification of practices in professional disciplines, interdisciplinary experiences in public health service, multidisciplinary practices and interventions in Primary Care. To promote advances in the vocational training process, it is necessary to create teaching-learning continuous strategies and to plan practices settings integrated with health services.

Keywords: Human resources in health training. Physioterapy. National health programs.
Este artigo buscou identificar, a partir da óptica de docentes e estudantes, ações que evidenciam a reorientação da formação profissional em Fisioterapia, no âmbito do eixo Cenários de Prática, conforme preconizado pelo Programa Pró-Saúde. Trata-se de uma pesquisa qualitativa que seguiu o método de estudo de caso, realizada em uma universidade comunitária com 16 estudantes e 11 docentes do curso de Fisioterapia. A coleta de dados foi conduzida por grupo focal e entrevista. Entre as ações que evidenciam a reorientação da formação profissional estão: realização do diagnóstico situacional, práticas em complexidade crescente, diversificação de práticas nas disciplinas profissionalizantes, vivências interdisciplinares no serviço público de saúde, práticas multiprofissionais e intervenções na Atenção Básica. Para promover avanços no processo de formação profissional, é necessário criar estratégias de ensino-aprendizagem contínuas e planejar as práticas de forma integrada com os serviços de saúde.

Palavras-chave: Capacitação de recursos humanos em saúde. Fisioterapia. Programas nacionais de saúde.

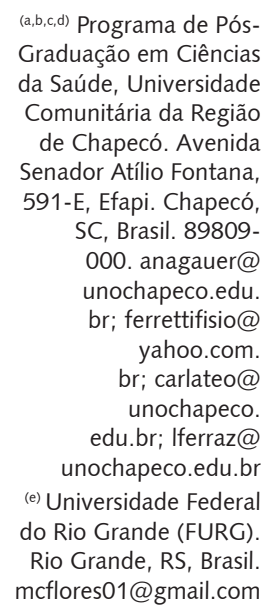

(a,b,c,d) Programa de PósGraduação em Ciências da Saúde, Universidade Comunitária da Região de Chapecó. Avenida Senador Atílio Fontana, 591-E, Efapi. Chapecó, SC, Brasil. 89809000. anagauer@ unochapeco.edu. br; ferrettifisio@ yahoo.com. br; carlateo@ unochapeco. edu.br; Iferraz@ unochapeco.edu.br (e) Universidade Federal do Rio Grande (FURG). Rio Grande, RS, Brasil. mcflores01@gmail.com 


\section{Introdução}

Na última década, os modelos pedagógicos que dão suporte à formação dos profissionais da área de saúde no País vêm sofrendo modificações devido à necessidade do desenvolvimento de competências e habilidades para uma atuação de acordo com os princípios do Sistema Único de Saúde (SUS $)^{1,2}$. Nessa perspectiva, a partir de 2005, as discussões sobre esse processo foram intensificadas e, em uma ação conjunta, os Ministérios da Educação e da Saúde deram início à criação de dispositivos para reorientar a formação profissional; entre eles, o Programa Nacional de Reorientação da Formação Profissional em Saúde (Pró-Saúde) e o Programa de Educação pelo Trabalho para a Saúde (PETSaúde) $)^{3,4}$.

Esses dispositivos valorizam o conhecimento para o SUS e a interação ensino-serviço; apresentamse como possibilidade de desvincular a formação centrada na assistência individual para uma mais direcionada à coletividade e às necessidades sociais, históricas, econômicas e culturais da população. Visam, ainda, superar a fragmentação dos modelos curriculares, formando profissionais que atentem para os determinantes do processo saúde-doença em todos os níveis³.

A estratégia proposta pelo Pró-Saúde organiza a reorientação da formação em três eixos: a orientação teórica, os cenários de prática e a orientação pedagógica. O foco deste estudo é o eixo Cenários de Prática, identificados como espaços de vivência no campo que permitem aos estudantes novos olhares, aproximação concreta com a realidade, integração com os profissionais do serviço, aproximação e intervenções na comunidade ${ }^{5,3}$. O eixo Cenários de Prática é dividido em três vetores: interação ensino-serviço, diversificação dos cenários do processo de ensino e articulação dos serviços universitários com o SUS ${ }^{3}$.

Dessa forma, torna-se importante compreender como vem avançando a formação profissional em Fisioterapia, tendo como base as estratégias preconizadas pelo Pró-Saúde, a fim de instrumentalizar a construção do conhecimento, a reflexão e o planejamento em relação à formação desses profissionais. Considerando o contexto apresentado, emerge a questão-problema deste estudo: Quais ações evidenciam o processo de reorientação da formação profissional de um curso de Fisioterapia nos cenários de prática?

Assim, considerando a inserção do curso de Fisioterapia da instituição estudada no Pró-Saúde, desde 2008, e no PET-Saúde, desde 2009, este estudo teve o objetivo de identificar, a partir da óptica de docentes e estudantes, ações que evidenciam a reorientação da formação profissional em Fisioterapia, no âmbito do eixo Cenários de Prática preconizado pelo Pró-Saúde.

\section{Metodologia}

Trata-se de estudo com abordagem qualitativa, orientado pelo método de estudo de caso6. A pesquisa foi realizada em um curso de graduação em Fisioterapia criado em 2004, em uma universidade comunitária. O curso se encontra inserido no Pró-Saúde e nos projetos do PET-Saúde desde 2008.

Participaram do estudo 16 estudantes que cursavam o sexto e o oitavo semestres do curso de Fisioterapia na época da pesquisa; cinco deles foram bolsistas do Pró-Saúde ou do PET-Saúde nos anos de 2014 e 2015. Além dos estudantes, 11 docentes fisioterapeutas também integraram a população de estudo.

Para a coleta de dados, foram realizados três grupos focais, dois com estudantes e um com docentes. Cada um dos grupos focais com estudantes contou com a participação de seis e dez discentes e o grupo focal realizado com docentes teve a participação de nove professores.

Para ampliar a compreensão do caso em estudo, foi utilizada também a técnica de entrevista semiestruturada com o docente articulador do Pró-Saúde no curso e com o coordenador pedagógico. Tanto as entrevistas quanto os grupos focais foram realizados conforme preconizado por Minayo ${ }^{7}$, seguindo um roteiro de questões pré-elaboradas sobre as ações realizadas no curso que evidenciam a reorientação da formação profissional, de acordo com o documento que regulamenta o Pró-Saúde 
no eixo Cenários de Práticas ${ }^{3}$. Os roteiros eram compostos por perguntas sobre a organização das práticas do curso, os cenários utilizados, como se davam as práticas nos serviços públicos de saúde e, especificamente, na Atenção Básica (AB), como eram organizadas as supervisões, se existiam ações interdisciplinares ou intercursos e a relação dos serviços próprios da instituição de ensino superior (IES) com o SUS.

Todos os participantes do estudo foram caracterizados quanto à idade; e os docentes, quanto ao tempo de graduação e titulação acadêmica. As entrevistas e o debate dos grupos focais foram gravados e, posteriormente, transcritos. O material textual passou por análise de conteúdo temática, realizada em três etapas - pré-análise; exploração do material; e tratamento dos resultados obtidos e interpretação -, conforme proposição de Minayo ${ }^{7}$.

O projeto foi aprovado pelo Comitê de Ética em Pesquisa da Instituição em que o estudo foi realizado, conforme resolução n. 466\12 do Conselho Nacional de Saúde. A fim de garantir a privacidade e preservar a identidade dos sujeitos, foram utilizados códigos para identificação das falas: E para estudantes, EB para estudantes bolsistas e D para docentes.

\section{Resultados e discussão}

A faixa etária dos estudantes variou de 19 a 22 anos de idade; dos docentes, de 25 e 44 anos. $\mathrm{O}$ tempo de formação dos professores variou entre seis e 21 anos. Em relação à titulação dos docentes, um era doutor; oito eram mestres e dois eram especialistas.

Após análise de conteúdo, emergiram seis categorias analíticas de ações que evidenciam a reorientação da formação profissional nos cenários de prática do curso de Fisioterapia estudado. Estas serão apresentadas na sequência.

\section{1) Realização do diagnóstico situacional}

Entre as ações desenvolvidas no curso que evidenciam a reorientação da formação profissional nos cenários de prática, identificamos a "realização do diagnóstico situacional" nas disciplinas relacionadas à saúde coletiva como elemento organizador das práticas em saúde, conforme relatos a seguir:

“Na disciplina de Saúde Pública, os estudantes do terceiro período foram para as unidades básicas de saúde, com o objetivo de reconhecer o espaço, pensar e visualizar o fisioterapeuta, trabalharam com conceito de saúde neste território". (D1)

“Na disciplina de Promoção da Saúde, no quinto período, se faz um primeiro diagnóstico e um reconhecimento do território. Posteriormente, realizam o planejamento de intervenções práticas". (D8)

O diagnóstico situacional é uma ferramenta utilizada para analisar o ambiente de uma determinada população ou serviço e pode contribuir para a identificação dos problemas ou necessidades que demandam aquele espaço ${ }^{8}$. Reconhecer o território é um dos passos para caracterizar uma determinada população e seus problemas de saúde, além de dar subsídios para avaliar o impacto dos serviços de saúde em seus diversos níveis?.

Os achados do estudo mostram que os estudantes vão ao cenário de prática e realizam um levantamento prévio da situação, vulnerabilidades e doenças prevalentes, para posteriormente elaborarem estratégias de intervenção, mas não realizam as práticas juntamente com os profissionais da saúde da Estratégia de Saúde da Família (ESF), dos Núcleos de Apoio à Saúde da Família (NASF), ou com os líderes comunitários daquele território, como pode ser observado a seguir em alguns relatos dos estudantes: 
“Nas disciplinas de Promoção da Saúde I e II, que são no quarto e quinto período, reconhecemos o território e traçamos o perfil da população que vamos intervir". (E8)

"Inicialmente, visitamos o espaço, porém, não foi feito um estudo aprofundado quando fomos para a $\operatorname{Adevosc}^{\left({ }^{()}\right.}$, não estudamos a instituição e o entorno antes de entrar lá". (EB1)

Esses relatos indicam a necessidade de se assumir o compromisso de estender as práticas de ensino nos diferentes serviços de saúde e articular com os profissionais e a comunidade, para, assim, promover a desfragmentação dos saberes. Para além do diagnóstico situacional, a territorialização(g) pode se constituir como um elemento organizador das práticas; possibilitar a interação do estudante com o território, com os problemas de saúde e com a comunidade; e estabelecer relações horizontais com outros setores e serviços, na perspectiva da intersetorialidade.

Pesquisa que buscou compreender a organização dos estágios em saúde coletiva sob a óptica dos preceptores e demonstrou que as intervenções se organizavam, primeiramente, com as discussões sobre o território, os equipamentos sociais e determinantes sociais da saúde. Os autores concluíram que a territorialização é considerada uma ferramenta que potencializa a integração ensino-serviço de saúde ${ }^{11}$.

Pondera-se que é preciso avançar no sentido de produzir e incorporar a territorialização nas ações pedagógicas, pois observamos nos relatos dos sujeitos que o processo ainda é reduzido ao diagnóstico situacional. Tanto a territorialização quanto o diagnóstico situacional contribuem para fortalecer a interação ensino-serviço por meio da inserção do estudante nos serviços de saúde e do estreitamento das relações com profissionais e comunidade, o que aproxima as práticas em saúde da realidade, produzindo tomadas de decisões mais eficazes.

\section{2) Organização das práticas em complexidade crescente}

Outra ação identificada é a "organização das práticas em complexidade crescente", principalmente aquelas previstas por meio de um programa instituído na matriz curricular do curso de Fisioterapia denominado Vivências. Esse programa organiza ações integradoras que ocorrem a cada semestre, em complexidade crescente, e que envolvem a observação, a avaliação, o acompanhamento e a intervenção nos três níveis de atenção em saúde, nas diversas áreas de conhecimentos fisioterapêuticos ${ }^{12}$. Algumas falas representam aspectos das práticas em complexidade crescente:

"As práticas articuladas ao Programa Vivências do curso começam no primeiro semestre, com a observação, até chegar às práticas assistidas". (E6)

“Os primeiros semestres têm 'O ser fisioterapeuta', em que nós vamos para as clínicas de Fisioterapia e observamos o fazer desse profissional no cotidiano". (E15)

“Nas disciplinas de Promoção da Saúde I e II (articuladas às Vivências IV e $\mathrm{V}$ ) realizamos as intervenções ainda no $\mathrm{CCl}^{(\mathrm{h})}$, Nosso Lar ${ }^{(\mathrm{i})}$, Adevosc, Apae $^{(j)}$, no CAPP(k) e no albergue municipal"(l). (EB3)

\begin{abstract}
(†) A Associação de Deficientes Visuais de Santa Catarina. $O$ curso realiza atividades que visam à promoção da saúde por meio de orientações e programa de exercício físico.
\end{abstract}

\footnotetext{
(g) Objetiva reconhecer o ambiente, a população e a dinâmica social existente nesse espaço por meio da identificação de aspectos geográficos e culturais do ambiente, do perfil demográfico, epidemiológico, político e social, além do mapeamento da área e do planejamento intersetorial ${ }^{10}$.

(h) Centro de Convivência de Idosos. São realizadas atividades com idosos institucionalizados.

(i) A Associação Espírita Nosso Lar objetiva auxiliar e capacitar gestantes carentes para confecção de enxovais aos seus filhos. São realizadas intervenções com as gestantes.

(j) Associação de Pais e Amigos dos Excepcionais. O curso realiza práticas de observação até a prática assistida nesse espaço.

(k) Centro de Atividades Psicossociais Patrick. O curso realiza práticas de observação até a prática assistida nesse espaço.

(I) O Albergue João Piltz oferece hospedagem e refeições às pessoas que fazem tratamento de oncologia ou que vêm à procura de uma vida melhor. O curso realiza intervenções com os acolhidos nesse espaço.
} 
As atividades teórico-práticas do Programa Vivências estão organizadas por semestres. No primeiro semestre, são previstas atividades de observação sobre o Ser Fisioterapeuta em diferentes contextos - clínicas, organizações e consultórios fisioterapêuticos -, que são registradas em diário de campo. Na segunda fase, são realizadas novas observações nesses espaços, mas os estudantes refletem sobre o Ser e o Fazer do Fisioterapeuta. Em uma terceira etapa, articulada com o Vivências Interdisciplinares e Multiprofissionais (VIM), projeto proposto pelo Pró-Saúde, são realizadas vivências na Atenção Básica à Saúde - o que será abordado na sequência. Na quarta e quinta fases, são realizadas práticas em que o foco é a atuação do fisioterapeuta na promoção da saúde em grupos populacionais específicos e em espaços como os mencionados no relato do sujeito EB3. Na sexta fase, os estudantes realizam avaliação e diagnóstico das necessidades de uma família e propõem intervenção. Na sétima fase, vivenciam o fazer do profissional, em outros estados e municípios, a fim de conhecer a realidade de trabalho do fisioterapeuta em diferentes contextos.

A vivência está organizada dentro de uma carga horária de quarenta horas, que representa dois créditos em cada semestre. Os relatos dos sujeitos do estudo demonstram que, no que se refere à necessidade de potencializar o aprendizado prático durante a formação desde o início do processo e em complexidade crescente ${ }^{3}$, o que está preconizado no documento do Pró-Saúde no eixo Cenários de Prática é atendido no curso.

Estudo realizado em um curso de Fisioterapia na Paraíba observou que os estudantes reconhecem a importância de processos formativos que estimulem a diversidade de ações de aprendizagem capazes de provocar mudanças nos conhecimentos e na formação ${ }^{13}$. Como destaca o sujeito D11, a seguir, há diversidade de vivências e locais diferentes para as práticas ao longo do processo de formação no curso em questão, viabilizado pelo Programa Vivências:

\footnotetext{
"No quarto e quinto período eles também se inserem em cenários práticos em populações diversas de acordo com o ciclo da vida, como crianças, mulheres, idosos, deficientes visuais, que são em espaços parceiros da universidade, todos em Chapecó [...]. No sétimo período, na disciplina de Estágio de Fisioterapia Geral, os estudantes vão para espaços fora do município, para que o estudante [...] vivencie esse espaço e também um pouco mais do trabalho do fisioterapeuta em outras realidades". (D11)
}

As diversificações das experiências garantem ao estudante o desenvolvimento da compreensão do trabalho a ser realizado nos diversos níveis de atuação. No curso estudado, têm sido ofertados alguns cenários de práticas para além da clínica privada, o que evidencia um compromisso com uma formação generalista e pautada em vivências extramuros, que fortalecem a construção de um perfil profissional embasado na integralidade da ação.

As práticas em contextos reais possibilitam aos estudantes compreender a dimensão do cuidado e os colocam como participantes do processo de trabalho em saúde, permitindo que adquiram habilidade para intervir em variadas situações, muitas delas oportunizadas pela diversidade dos cenários. Assim, as práticas instrumentalizam os estudantes para a atuação profissional em suas diferentes áreas de atuação ${ }^{14}$.

A diversidade de experiências ao longo da formação promove o desenvolvimento de estratégias de enfrentamento saudáveis mediante os desafios da profissão, além de potencializar processos de ensino-aprendizagem que enriquecem a relação teoria-prática ${ }^{15}$. Além da diversidade de experiências descritas anteriormente, as práticas em complexidade crescente possibilitam ao estudante construir habilidades de acordo com o grau de autonomia desenvolvido ao longo do curso.

\section{3) Vivências práticas interdisciplinares}

É importante pontuar que, além das práticas em complexidade crescente, as vivências práticas interdisciplinares também foram elencadas pelos sujeitos do estudo como atividades que evidenciam a reorientação da formação profissional no curso. A interdisciplinaridade pode ser compreendida como "[...] a integração entre as disciplinas e a intensidade de trocas entre os especialistas; desse processo interativo, todas as disciplinas devem sair enriquecidas"15 (p. 109). 
As vivências práticas interdisciplinares do curso são oferecidas principalmente pelo Projeto Vivências Interdisciplinares e Multiprofissionais (VIM), destacado por estudantes e professores como uma prática importante, da qual o curso de Fisioterapia participa junto com os demais cursos da área da saúde:

"O VIM no terceiro período [...] somos distribuídos com estudantes dos outros cursos [...] e vamos a unidades básicas de saúde reconhecer território".

“No VIM vamos reconhecer a atuação de cada profissional dentro da unidade básica de saúde". (E8)

"O programa realiza atividades com estudantes e professores de outros cursos. Eu acredito que isso impactou positivamente em todos, pelo menos no meu grupo conseguimos discutir sobre vários casos dos serviços, com a ideia de cada área, de acordo com sua futura profissão". (E11)

O VIM foi elaborado a partir da proposta do Pró-Saúde, fruto de um processo de discussão dos professores da área de Ciências da Saúde e dos gestores da Secretaria Municipal de Saúde. O projeto VIM tem como objetivo a inserção dos estudantes na $A B$ com o intuito de observar as atividades realizadas no local, interagindo com profissionais e usuários do serviço. Esse projeto envolve estudantes dos cursos das áreas da saúde e ciências biológicas e das ciências sociais e humanas dessa IES, tendo como tutores profissionais dos serviços públicos de saúde, além de docentes dos cursos envolvidos ${ }^{16}$.

Pesquisa com estudantes da área da saúde que realizavam ações interdisciplinares junto com a equipe de saúde evidenciou a importância desse trabalho para o alcance da integralidade das ações no âmbito da Atenção Básica ${ }^{17}$, constituindo-se em uma experiência singular que potencializa a formação profissional.

Uma formação direcionada para o trabalho em equipe transforma as práticas em saúde e as direciona para a integralidade no cuidado, além de possibilitar aos profissionais um melhor planejamento das ações, sem se restringir ao processo saúde-doença ${ }^{18,19}$.

Segundo o documento do Pró-Saúde, a atuação interdisciplinar e o trabalho em equipes multiprofissionais são pouco explorados pelas IES, o que se assemelha nas equipes de saúde, tendo como consequência as ações isoladas de cada profissional e a fragmentação do cuidado ${ }^{3}$. Nesse sentido, as práticas interdisciplinares precisam ser estimuladas, visto que instigam a troca de saberes e principalmente a criação de estratégias que melhoram as intervenções em saúde.

\section{4) Práticas multiprofissionais}

Outra ação de reorientação destacada pelos sujeitos do estudo foi a realização de práticas multiprofissionais, conforme verificado nestes depoimentos:

"O estágio de oncologia desenvolve uma prática, há três anos, com três disciplinas do curso de Psicologia. Fazem atendimento em grupo com os pacientes da clínica-escola de Fisioterapia. São atividades coletivas nas quais eles participam e fazem devolutiva com os estudantes de Fisioterapia. Tem ainda a avaliação e atendimento psicológico, essa ação é mais pontual". (D7)

"No quarto e quinto período, as disciplinas de promoção da saúde têm estimulado a convidar outros estudantes a se inserirem nas atividades da Fisioterapia, já tivemos atividades bem interessantes com os estudantes da Nutrição, Psicologia e Educação Física, que realizavam as práticas de promoção da saúde na comunidade conosco, após nosso convite". (D11)

A prática multiprofissional é considerada uma modalidade de trabalho coletivo que busca a articulação de diversas disciplinas, e cada profissional atuará de acordo com o seu saber especializado $^{19}$. Esse modelo de trabalho emerge da necessidade de efetivar o cuidado integral em 
saúde como umas das estratégias de reorganização dos serviços de saúde, focada na prática integrada entre as diversas profissões ${ }^{20}$. $O$ trabalho multiprofissional deve ser desempenhado por vários profissionais e é a base para avançar na interdisciplinaridade.

Para tanto, emerge a necessidade de inserir as atividades multiprofissionais nos currículos, de modo que o estudante tenha conhecimento e preparo para atuar em equipe ${ }^{21}$. No processo de organização do curso, têm sido observados esforços para a realização de ações multiprofissionais por meio do incentivo a essas ações nos estágios supervisionados vinculados à saúde coletiva, conforme relato:

“No grupo de incontinência urinária que realizamos no Centro de Saúde do BV, as atividades ocorrem em conjunto com o curso de Medicina, que está no internato de Ginecologia e Obstetrícia [...]. Dentro do estágio já foi feito algumas vezes o dia do tabaco [...] a Nutrição foi falar sobre o que acontecia com os receptores gustativos; a Odontologia foi falar um pouco da oralidade, da higiene bucal; a Fisioterapia, das questões da fisioterapia respiratória; a Educação Física trabalhou com a importância da prática de atividade física; a Medicina trabalhou com a temática das doenças prevalentes; e a Psicologia, o instrumento emocional do cigarro [...]. Foi uma atividade pontual, claro, incipiente, mas temos tentado [...]". (D11)

Estimular essas práticas multiprofissionais ao longo do processo de formação amplia a visão de cuidado do sujeito e busca o desenvolvimento de competências que colaboram para o cuidado do sujeito de maneira integral, previstas como necessidade da formação profissional para o SUS.

Relato de experiência de uma disciplina que detalhou a realização de atividades nos serviços do SUS em um contexto multiprofissional, a partir do olhar dos docentes e estudantes, concluiu que a proposta possibilitou aos estudantes convívio, troca de experiências e produção coletiva textual, consolidandose como um processo permanente de qualificação e atuação para profissionais, estudantes e professores $^{21}$.

Nesta perspectiva, potencializar e programar essas ações depende do planejamento pedagógico, acompanhamento e gestão do curso. Há necessidade de se planejar e integrar mais ações multiprofissionais, como uma estratégia permanente no processo de formação, diversificando os espaços e realizando ações em conjunto com as equipes da ESF e NASF. Torna-se importante, para avançar, planejar ações multiprofissionais no serviço e na comunidade, articulando a IES com NASF e ESF para integrar profissionais, estudantes e professores.

Essas experiências multiprofissionais têm incentivado a ampliação do processo de formação dos profissionais da saúde, direcionando para a ruptura do ensino em especialidades, por meio de uma intervenção ampliada e da produção de novos saberes, tendo como consequência uma melhora no atendimento dos serviços. Para o ensino, as ações multiprofissionais precisam ser permanentes e contínuas, incorporadas aos planos de ensino e ao planejamento dos cursos.

\section{5) Diversificação de práticas nas disciplinas profissionalizantes}

Também foi descrita pelos atores deste estudo como uma ação de reorientação da formação a diversificação de práticas nas disciplinas profissionalizantes da Fisioterapia, conforme relatos:

"Na disciplina de Fisioterapia em Ergonomia, no quinto período, realizamos as práticas nos setores da universidade, em pequenos grupos. Realizamos ações ergonômicas e de atividade laboral, e posteriormente elaboramos estratégias para melhoria no setor [...]".

"Nas áreas da ortopedia e desportiva realizamos as intervenções com os atletas no laboratório de fisioterapia, isso ocorreu no quinto e sexto período [...], na disciplina de Fisioterapia na Geriatria e Gerontologia, vamos aos centros de convivência de idosos, na disciplina de Fisioterapia Respiratória, vamos para o hospital; ambas ocorrem no sexto período". (E16) 
“Na área da cardiorrespiratória, as práticas ocorrem aqui no laboratório, na clínica-escola e no hospital regional, são organizadas avaliação e intervenção com pacientes". (D9)

A diversificação dos espaços de práticas nas áreas profissionalizantes da formação do fisioterapeuta possibilita o contato com usuários e familiares e a efetivação da articulação da teoria com a prática, contribuindo para superar a fragmentação dos saberes.

São diversas as vivências práticas em disciplinas profissionalizantes socializadas pelos sujeitos deste estudo:

\footnotetext{
“No componente de Fisioterapia em Geriatria e Gerontologia, neste semestre, fizemos um trabalho de campo, em grupos de idosos na comunidade e em centros comunitários, interessante é que foram para espaços tanto urbanos como rurais". (D1)

"Dentre os locais de práticas [...] temos atendimentos da comunidade geral e em alguns salões comunitários que estão vinculados às unidades básicas de saúde". (D10)
}

A comunidade pode ser um campo fértil de ensino, possibilitando ao estudante praticar no cenário e articular as atividades naquele contexto, o que pode viabilizar a integração das práticas com equipamentos sociais presentes na comunidade como escolas, grupos comunitários e equipes de ESF $^{22}$. Estudo realizado com egressos do curso de Odontologia observou a importância dos estágios na comunidade para formação: essas experiências representaram para o grupo de estudantes um espaço de reflexão sobre as ações em saúde ${ }^{20}$.

Para além disso, práticas na comunidade proporcionam o exercício da responsabilidade social, permitindo aquisição de conhecimentos e desenvolvimento de habilidades e atitudes, adquiridas no contato direto com diferentes atores que convivem nesses espaços ${ }^{23}$. Dessa forma, as práticas em diversos cenários devem integrar as áreas básicas e profissionalizantes, ao longo de todo o curso, para garantir uma formação generalista, que atue nos três níveis de atenção em saúde.

\section{6) Intervenções na Atenção Básica à Saúde}

Também na óptica dos docentes e estudantes, as intervenções na Atenção Básica à Saúde foram identificadas como ações que evidenciam a reorientação:

“Os estágios de saúde coletiva desenvolvem práticas na Atenção Básica com grupo de gestantes, incontinência urinária, ginástica laboral com as agentes comunitárias de saúde, intervenção na sala de espera e as visitas domiciliares". (D2)

A Atenção Básica à Saúde pode ser definida como um conjunto de ações de saúde, nos âmbitos individual e coletivo, que envolve a promoção e a proteção da saúde, a prevenção de agravos, o diagnóstico, o tratamento, a reabilitação e a redução de danos por intermédio do trabalho em equipe, dirigidos a populações de territórios definidos ${ }^{24}$. A Atenção Básica é a principal porta de entrada do SUS e possibilita resolver grande parte dos problemas de saúde, evitando a busca pelo atendimento nas emergências dos hospitais.

É necessário incentivar as ações na Atenção Básica desde o início da formação, fortalecendo a relação entre teoria e prática e oportunizando aos estudantes um espaço de construção do conhecimento, em atuação articulada com o serviço ${ }^{25}$.

No curso estudado, apesar de haver a previsão de realização de práticas na Atenção Básica, elas ocorrem, especialmente, nos últimos anos do curso. Nesse aspecto, há necessidade de avanços, para que essa inserção busque uma articulação das atividades do ensino com a rotina de trabalho da equipe e o planejamento de ações com os profissionais do serviço. Essas ações, quando ocorrem de forma pontual dentro dos serviços, não são suficientes para provocar de fato mudanças na formação para o SUS ${ }^{3}$. 
Estudo realizado com estudantes do curso de Medicina em uma universidade de Minas Gerais evidenciou que eles reconheceram a Atenção Básica como um cenário positivo para o processo de formação, pois a inserção produz uma relação mais próxima com os usuários e o conhecimento da complexidade do SUS. O estudo destacou, ainda, a importância da realização de visitas domiciliares como práticas de estágio ${ }^{26}$. Docentes e estudantes apontaram, nesse estudo, a realização das visitas domiciliares como uma das ações realizadas na $A B$, conforme fala de D2 apresentada anteriormente.

A visita domiciliar é uma das atividades realizadas pelos profissionais que atuam na Atenção Básica. Estudo que buscou conhecer os significados atribuídos à visita domiciliar na ótica de estudantes de Fisioterapia destacou, dentre os principais achados, que a vivência na realidade das famílias atendidas colaborou com o desenvolvimento de habilidades e competências necessárias ao trabalho em saúde, o que fortalece a construção do perfil profissional do fisioterapeuta ${ }^{6,27}$.

Em relação às intervenções na Atenção Básica, neste estudo, foram relacionadas somente as disciplinas vinculadas à saúde pública, conforme estes relatos:

"A disciplina de Fisioterapia na Atenção Básica e também o Estágio de Saúde Coletiva, que realmente faz práticas na Atenção Básica". (D10)

“No Estágio de Saúde Coletiva, a primeira atividade quando eles chegam ao território [...] participam de uma reunião da equipe para entender também o fluxo, demanda, os obstáculos do trabalho, reconhecem o entorno passeiam em torno das áreas a que aquele espaço pertence, para posteriormente realizar o estágio obrigatório com a comunidade e profissionais do serviço". (D11)

Esses relatos demonstram que o curso estudado apresenta uma diversidade de práticas em diferentes cenários, mas necessita avançar e garantir melhores possibilidades de práticas nos serviços do SUS. O curso pode integrar as ações de nível primário, secundário e terciário nesses espaços públicos, visto que "[...] a rede básica não elimina os níveis mais complexos, sendo que as linhas de cuidado (da promoção à recuperação) representam a estratégia do SUS para enfrentar o enorme desafio de otimizar os recursos disponíveis nesta proposta ousada que é um sistema universal e equânime" ${ }^{3}$.

Pesquisa realizada em uma universidade da Bahia com estudantes do curso de Fisioterapia sobre sua percepção em relação à atuação na Atenção Básica à Saúde evidenciou que os estudantes referiram não se sentirem aptos a atuar nesse espaço quando formados, o que representa uma fragilidade durante o processo de ensino-aprendizagem do fisioterapeuta nesse âmbito. Segundo os autores, isso se deu devido ao fato de a construção das práticas nessa área ocorrer de forma limitada a poucas disciplinas, principalmente nas últimas fases do $\mathrm{CursO}^{27}$. Aquela realidade se assemelha à do curso ora estudado.

As ações na $A B$ são realizadas no estágio supervisionado de saúde coletiva e também no componente curricular de Fisioterapia na Saúde Pública, que articula o projeto VIM, referido anteriormente. Práticas pontuais podem não ser suficientes para propor uma mudança na formação; fazem-se necessários mecanismos que proponham o incentivo do cuidado em uma abordagem integral do processo saúde-doença, por intermédio da inserção dos estudantes no cenário real do SUS.

Diante das ações que evidenciam a reorientação da formação, o curso em questão, no que se refere à interação ensino-serviço, em nossa análise, precisa avançar, pois conta com alguma articulação da programação teórica com a prática dos serviços públicos de saúde, mas em poucas áreas disciplinares ${ }^{3}$. No caso do curso, essa articulação ocorre em disciplinas vinculadas à saúde pública, com pouca interação das áreas disciplinares; a instituição como um todo não integra durante o processo de ensino a orientação teórica com a prática nos serviços públicos de saúde, principalmente na Atenção Básica, com a participação de todas as áreas disciplinares.

A diversificação dos cenários do processo de aprendizagem também precisa melhorar, visto que propõe atividades extramurais isoladas de acadêmicos em unidades do SUS, durante os primeiros anos do curso, com a participação predominante de professores da área de Saúde Coletiva, 
correspondendo a um pequeno percentual da carga horária semanal do aluno. Além disso, o ciclo clínico é majoritariamente baseado em atividades assistenciais em instalações da universidade não funcionalmente vinculadas ao SUS ${ }^{3}$.

Quanto à articulação dos serviços universitários com o SUS, o curso está avançando, pois prevê serviços parcialmente abertos ao SUS, preservando algum grau de autonomia na definição dos pacientes a serem atendidos - os atendimentos são por demanda reprimida do SUS. Para alcançar satisfatoriamente a interação ensino-serviço e a diversificação dos cenários do processo de aprendizagem, o curso precisa construir estratégias que articulem as práticas nas diversas áreas disciplinares por meio das evidências propostas pelo documento do Pró-Saúde, em que os parâmetros sugeridos são o envolvimento da comunidade como espaço social participativo, incluindo o cuidado de saúde em todos os níveis de atenção, com a orientação coletiva, a participação de toda a instituição e o ajuste do cronograma do curso à lógica dos serviços. A meta a ser alcançada descreve, ainda, as práticas de atenção à saúde, ao longo de todo o curso, com carga horária nos diferentes níveis de atenção ${ }^{3}$.

Nessa direção, é necessário propor um diálogo com o serviço e integrar o espaço com o SUS. Isso porque o espaço físico já existe; assim, a necessidade é a de que este seja melhor aproveitado e que os recursos humanos da IES possam atuar nesse espaço segundo as premissas do SUS.

\section{Considerações finais}

Ao buscar identificar ações que evidenciem a reorientação da formação profissional em Fisioterapia, no âmbito do eixo Cenários de Prática, o estudo evidencia que as atividades realizadas aproximam o ensino do serviço e da comunidade. Mostra também que há uma organização das práticas em complexidade crescente no curso estudado, com incentivo para realização dessas vivências em diferentes realidades de saúde, desde o primeiro ano, o que tem promovido a diversificação dos cenários de aprendizagem.

Para formar profissionais preparados para atender as demandas de saúde do SUS, é necessário fortalecer a interação do ensino com o serviço e a inserção de práticas em complexidade crescente na Atenção Básica $(A B)$ e nos serviços públicos de saúde, com ações interdisciplinares e que garantam ao estudante uma diversidade de práticas, com ações em todos os níveis de assistência. Construir estratégias de ensino-aprendizagem contínuas e planejar as atividades conjuntamente com os profissionais do serviço são ações que o curso de graduação em Fisioterapia precisa desenvolver para avançar no processo de reorientação da formação.

Há necessidade de realizar um movimento coletivo que estimule o diálogo e a maior integração entre os gestores da IES, do serviço e dos cursos da área da saúde. Torna-se necessário pactuar, organizar e negociar as inserções dos estudantes na $A B$, nas quais se dialogue sobre as necessidades do coletivo, não individualmente, curso a curso. Essas estratégias precisam ser planejadas de forma participativa, ouvindo os atores envolvidos no processo, para efetivamente produzir mudanças.

Como estratégia para que esses desafios sejam transpostos, a institucionalização das práticas do Pró/PET-Saúde pode ser um importante mecanismo, contribuindo para consolidar mudanças na formação profissional, já que as ações promovidas por esses dispositivos possibilitam a aproximação ensino-serviço com práticas interdisciplinares nos serviços públicos de saúde, principalmente na Atenção Básica, e ofertam uma diversificação de cenários para efetivar a relação teoria e prática. 


\section{Colaboradores}

Ana Paula Maihack Gauer, Fátima Ferretti e Carla Rosane Paz Arruda Teo participaram da elaboração do artigo na íntegra, redação, discussão e revisão do texto. Lucimare Ferraz e Maria Cristina Flores-Soares participaram da revisão e aprovação da versão final do trabalho.

\section{Referências}

1. Freitas PH, Colome JS, Carpes AD, Backes DS, Beck CLC. Repercussões do pet-saúde na formação de estudantes da área da saúde. Esc Anna Nery. 2013; 17(3):496-504.

2. Leal JAL, Melo CMM, Veloso RBP, Juliano IA. Novos espaços de reorientação para formação em saúde: vivências de estudantes. Interface (Botucatu). 2015; 19(53):361-71.

3. Ministério da Saúde (BR), Ministério da Educação (BR). Programa Nacional de Reorientação da Formação Profissional em Saúde - Pró-Saúde: objetivos, implementação e desenvolvimento potencial. Brasília, DF: Ministério da Saúde; 2007.

4. Ministério da Saúde (BR), Ministério da Educação (BR). Portaria Interministerial $n^{\circ}$ 1.802, de 26 de agosto de 2008. Institui o Programa de Educação pelo Trabalho para a Saúde-PET-Saúde. Diário Oficial União. 26 Ago 2008; Seç. 1:97.

5. Morais FRR, Jales GML, Silva MJC, Fernandes SF. A importância do PET-Saúde para a formação acadêmica do enfermeiro. Trab Educ Saude. 2012; 10(3):541-51.

6. Yin RK. Estudo de caso: planejamento e métodos. 4a ed. Porto Alegre: Bookmann; 2010.

7. Minayo MCS. O desafio do conhecimento: pesquisa qualitativa em saúde. 14a ed. São Paulo: Hucitec; 2014.

8. Luvisaro BMO, Lima GS, Freire EMR, Martinez MR. Diagnóstico situacional em unidade de terapia intensiva: relato de experiência. Rev Adm Hosp Inov Saude. 2014; 11(2):67-78.

9. Monken $M$, Barcellos $C$. Vigilância em saúde e território utilizado: possibilidades teóricas e metodológicas. Cad Saude Publica. 2005; 21(3):898-906.

10. Pereira MP, Barcellos C. O território no programa de saúde da família. Hygeia. 2006; 2(2):47-55.

11. Forte FDS, Pessoa TRRF, Freitas CHSM, Pereira CAL, Carvalho JPM. Reorientação na formação de cirurgiões-dentistas: o olhar dos preceptores sobre estágios supervisionados no Sistema Único de Saúde (SUS). Interface (Botucatu). 2015; 19 Suppl 1:831-43.

12. Universidade Comunitária. Projeto Pedagógico do Curso Estudado. Projeto Pedagógico do Curso de Graduação em Fisioterapia. Cidade; 2014.

13. Medeiros DKS, Neves RF. Análise crítica das práticas na atenção primária à saúde com base nos relatos dos estudantes do curso de fisioterapia. Rev Baiana Saude Publica. 2013; 37(1):87-105.

14. Benito GAV, Tristão KM, Paula ACSF, Santos MA, Ataide LJ, Lima RCD. Desenvolvimento de competências gerais durante o estágio supervisionado. Rev Bras Enferm. 2012; 65(1):172-8.

15. Costa RP. Interdisciplinaridade e equipes de saúde: concepções. Mental. 2007; 5(8):107-24.

16. Miechuanski PC, Kleba ME. Acadêmicos da Unochapecó na interação com sistema único de saúde e ESF através do Projeto Pró-Saúde. Rev Bras Educ Med. 2012; 36 Suppl 2:131-5. 
17. Alvarenga JPO, Meira AB, Fontes WD, Xavier MMFB, Trajano MP, Neto GC, et al. Multiprofissionalidade e interdisciplinaridade na formação em saúde: vivências de graduandos no estágio regional interprofissional. Rev Enferm UFPE. 2013; 7(10):5944-51.

18. Casanova IA, Batista NA, Ruiz-Moreno L. Formação para o trabalho em equipe na residência multiprofissional em saúde. ABCS Health Sci. 2015; 40(3):229-33.

19. Salvador AS, Medeiros CS, Cavalcanti PB, Carvalho RN. Construindo a multiprofissionalidade: um olhar sobre a residência multiprofissional em saúde da família e comunidade. Rev Bras Cienc Saude. 2011; 15(3):329-38.

20. Fernandes EGS, Masieiro AV, Kuhnen M. Percepção de cirurgiões dentistas inseridos na estratégia de saúde da família sobre o trabalho multiprofissional. Rev GepesVida. 2015; 1(2):56-70.

21. Toassi RFC, Lewgoy $A M B$. Práticas integradas em saúde I: uma experiência inovadora de integração intercurricular e interdisciplinar. Interface (Botucatu). 2016; 20(57):449-61.

22. Panúncio-Pinto MP, Rodrigues MLV, Fiorati RC. Novos cenários de ensino: a comunidade e o território como espaços privilegiados de formação de profissionais da saúde. Medicina (Ribeirão Preto). 2015; 48(3):257-64.

23. Brandão ERM, Rocha SV, Silva SS. Práticas de integração ensino-serviço-comunidade: reorientando a formação médica. Rev Bras Educ Med. 2013; 37(4):573-7.

24. Brehmer LCF, Ramos FRS. O modelo de atenção à saúde na formação em enfermagem: experiências e percepções. Interface (Botucatu). 2016; 20(56):135-45.

25. Massote AW, Belisário AS, Gontijo ED. Atenção primária como cenário de prática na percepção de estudantes de medicina. Rev Bras Educ Med. 2011; 35(4):445-53.

26. Medeiros PA, Pivetta HMF, Mayer MS. Contribuições da visita domiciliar na formação em fisioterapia. Trab Educ Saude. 2012; 10(3):407-26.

27. Silva LWS, Souza M, Souza TO, Souza TF. Contexto do cuidado fisioterapêutico: reveses e vieses na inserção comunitária à atenção domiciliar. Rev Kairós. 2013; 16(3):79-101.

Gauer APM, Ferretti F, Teo CRPA, Ferraz L, Soares MCF. Acciones de reorientación de la formación profesional en Fisioterapia: enfoque sobre escenarios de práctica. Interface (Botucatu). 2018; 22(65):565-76.

El objetivo de este artículo fue identificar, a partir de la óptica de docentes y estudiantes, acciones que dejan en evidencia la reorientación de la formación profesional en Fisioterapia, en el ámbito del eje Escenario de Práctica, conforme determinado por el programa Pro-Salud. Se trata de una investigación cualitativa que siguió el método del estudio de caso, realizada en una universidad comunitaria con 16 estudiantes y 11 docentes del curso de Fisioterapia. La colecta de datos se realizó por medio de grupo focal y entrevista. Entre las acciones que dejan en evidencia la reorientación de la formación profesional están las siguientes: realización del diagnóstico situacional, prácticas de complejidad creciente, diversificación de prácticas en las asignaturas profesionalizantes, vivencias interdisciplinarias en el servicio público de salud, prácticas multi-profesionales e intervenciones en la Atención Básica. Para promover avances en el proceso de formación profesional es necesario crear estrategias de enseñanza-aprendizaje continuas y planificar las prácticas de forma integrada con los servicios de salud.

Palabras clave: Capacitación de recursos humanos en salud. Fisioterapia. Programas nacionales de salud.

Submetido em 17/10/16. Aprovado em 01/02/17. 